\title{
Whole transcriptome sequencing identifies tumor-specific mutations in human oral squamous cell carcinoma
}

\author{
Qu Zhang ${ }^{1^{*}}$, Jun Zhang ${ }^{2}$, Hong Jin $^{3}$ and Sitong Sheng ${ }^{3^{*}}$
}

\begin{abstract}
Background: The accumulation of somatic mutations in genes and molecular pathways is a major factor in the evolution of oral squamous cell carcinoma (OSCC), which sparks studies to identify somatic mutations with clinical potentials. Recently, massively parallel sequencing technique has started to revolutionize biomedical studies, due to the rapid increase in its throughput and drop in cost. Hence sequencing of whole transcriptome (RNA-Seq) becomes a superior approach in cancer studies, which enables the detection of somatic mutations and accurate measurement of gene expression simultaneously.
\end{abstract}

Methods: We used RNA-Seq data from tumor and matched normal samples to investigate somatic mutation spectrum in OSCC.

Results: By applying a sophisticated bioinformatic pipeline, we interrogated two tumor samples and their matched normal tissues and identified 70,472 tumor somatic mutations in protein-coding regions. We further identified 515 significantly mutated genes (SMGs) and 156 tumor-specific disruptive genes (TDGs), with six genes in both sets, including ANKRA2, GTF2H5, STOML1, NUP37, PPP1R26, and TAF1L. Pathway analysis suggested that SMGs were enriched in cell adhesion pathways, which are frequently indicated in tumor development. We also found that SMGs tend to be differentially expressed between tumors and normal tissues, implying a regulatory role of accumulation of genetic aberrations in these genes.

Conclusions: Our finding of known tumor genes proves of the utility of RNA-Seq in mutation screening, and functional analysis of genes detected here would help understand the molecular mechanism of OSCC.

Keywords: RNA-Seq, Oral squamous cell carcinoma, Somatic mutations, Significantly mutated genes, Differential expression, Disruptive genes

\section{Background}

Squamous cell carcinoma is one of the most commonly observed cancers worldwide [1], which is often diagnosed in the oropharynx and oral cavity. It is highly invasive and metastatic at the advanced stage, and presents a substantial threat to human health [2]. Evidence from various molecular and genetic studies suggests an association between squamous cell carcinoma initiation and development and the accumulation of genetic alterations at both the DNA

\footnotetext{
* Correspondence: quzhang@post.harvard.edu; sst@hykgene.com 'Department of Human Evolutionary Biology, Harvard University, Cambridge, MA 02138, USA

${ }^{3} \mathrm{HYK}$ High-throughput Biotechnology Institute, 4th Floor, Building No.11, Software Park, 2nd Central Keji Rd, Hi-Tech Industrial Park, Shenzhen, China Full list of author information is available at the end of the article
}

and RNA levels [3]. Genomic alterations such as point mutations and copy number variations, epigenetic changes such as methylation and histone modifications, as well as gene expression changes have been previously revealed in oral squamous cell carcinoma (OSCC), which could facilitate biomarker development and make clinical decisions [3]. Among them, mutations only occurring in tumor tissues, often referred as somatic mutations, are given particular attention. It is widely accepted that tumors develop through the accumulation of somatic mutations in specific genes, depending on their types [4]. Various studies have found a higher than expected mutation frequency of candidate cancer genes, and that the tumor properties could be influenced by different combinations of mutations [5-8].

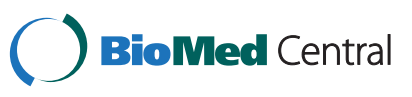


However, the high cost of Sanger sequencing prevents global profiling of somatic mutations in OSCC, and further understanding in mechanisms and clinical treatments.

Remarkable advances in sequencing technology over the last several years make possible to identify genetic alterations in a genome-wide scale. RNA-Seq is a newly developed deep sequencing technology, which is extensively applied in transcriptomic profiling due to its affordable cost. Compared with long standing methods such as microarray, RNA-Seq gives a far more precise measurement of transcript expression levels and a far more sophisticated characterization of transcript isoforms $[9,10]$. Therefore it has been successfully applied to identify differentially expressed genes [11] and to characterize allelespecific expression patterns [12,13]. Moreover, it is also an efficient and cost-effective way to study genomic alterations, such as somatic mutations in transcribed regions [14-17] or gene fusions [12-14]. Herein, we conducted a genome-wide study to investigate the somatic mutation spectrum in OSCC by interrogating RNA-Seq data from two tumor samples and their matched normal samples. We developed a sophisticated pipeline to identify somatic mutations, and then identified significantly mutated genes (SMG) and tumor-specific disruptive genes (TDG). By comparing with gene expression pattern, we also found a correlation between differentially expressed genes and SMGs. These findings demonstrate the ability of RNA-Seq to characterize global pattern of somatic mutations and suggest the potential mechanism on how somatic mutations could affect tumor development.

\section{Methods}

\section{Deep sequencing data}

Whole transcriptome short reads of three paired tumor and normal tissues from patients with oral squamous cell carcinoma (OSCC) were downloaded from European $\mathrm{Nu}-$ cleotide Archive (ENA, http://www.ebi.ac.uk/ena) with the accession number SRP002009. As described in the original paper, the study was conducted according to the Declaration of Helsinki, and was approved by the Institutional Review Board of the Mayo Clinic [18]. Written informed consent for the collection of samples and subsequent analysis was available for all patients. 50-bp sequence reads were generated by using Applied Biosystems SOLiD System (V3 chemistry), following the manufacturer's instructions. More details can be found in the original study [18].

\section{Sequence alignments}

We first excluded low quality reads in which one or a few bases have Q-score lower than 20. The initial quality check suggests that all reads from one patient have an average quality score $<20$ and were excluded from further analysis. Then qualified short reads were aligned to 18,462 transcripts of UCSC consensus coding sequences
(CCDS) in current human genome assembly (hg19, http://genome.ucsc.edu/). The alignment was carried out using BFAST [19], using options for color-space reads.

\section{Variant calling and identification of somatic variants}

We called variants from the SAM format read alignments using SAMtools package [20] for each sample. We first discarded alignments with the mapping quality lower than 30, and then made variant calls by mpileup and bcftools programs embedded in SAMtools [20]. To avoid potential PCR duplicate fragments, we discarded all variants covered by more than 500 reads, which was achieved by setting $-\mathrm{D}$ as 500 when invoking vcfutils.pl script. Several additional filters were also applied to minimize false positive rate (Figure 1):

Filter 1 Variants were removed if they are supported by less than two reads or mistakenly called with a probability greater than 0.01 . This was done by requiring a value $\geq 20$ for the 'QUAL' column in vcf files generated by SAMtools.

Filter 2 Somatic variants were called by comparing matched normal and tumor tissues. We first excluded variants located in genomic regions of poor quality, which were defined as regions with read coverage in only one of a sample pair, probably due to randomness in sequencing process.

Filter 3 Variants that are found in both of the matched normal and tumor samples were discarded. Also, variants found in dbSNP build 132 [21] were also excluded.

\section{Identification of significantly mutated genes}

To find significantly mutated genes, we adopted approaches implemented in MuSiC analysis tool suites [22]. Briefly, we counted the number of bases with at least three read depth in six categories, including $\mathrm{A}$ bases, $\mathrm{T}$ bases, $\mathrm{C}$ bases in $\mathrm{CpG}, \mathrm{G}$ bases in $\mathrm{CpG}, \mathrm{C}$ bases not in $\mathrm{CpG}$, and $\mathrm{G}$ bases not in $\mathrm{CpG}$. Then the discovered mutations were categorized as AT transitions, AT transversions, CpG transitions, $\mathrm{CpG}$ transversions, CG (non-CpG) transitions, CG transversions, and indels. Next, we calculated the background mutation rate (BMR) for each mutation category, which was done by dividing the total count of such category by the total number of available bases within such category. For indels, BMR is calculated as the total number of bases covered by indels divided by the total number of high quality bases. Since we used RNA-Seq data and the read depth depends on the expression level, tests that consider mutation coverage to identify significantly mutated genes may not be appropriate due to confounding factors such as allele-specific expression. Instead, we calculated the possibility of finding more mutations than the observation for each mutation category and combined then by the simple Fisher's combined $P$-value 


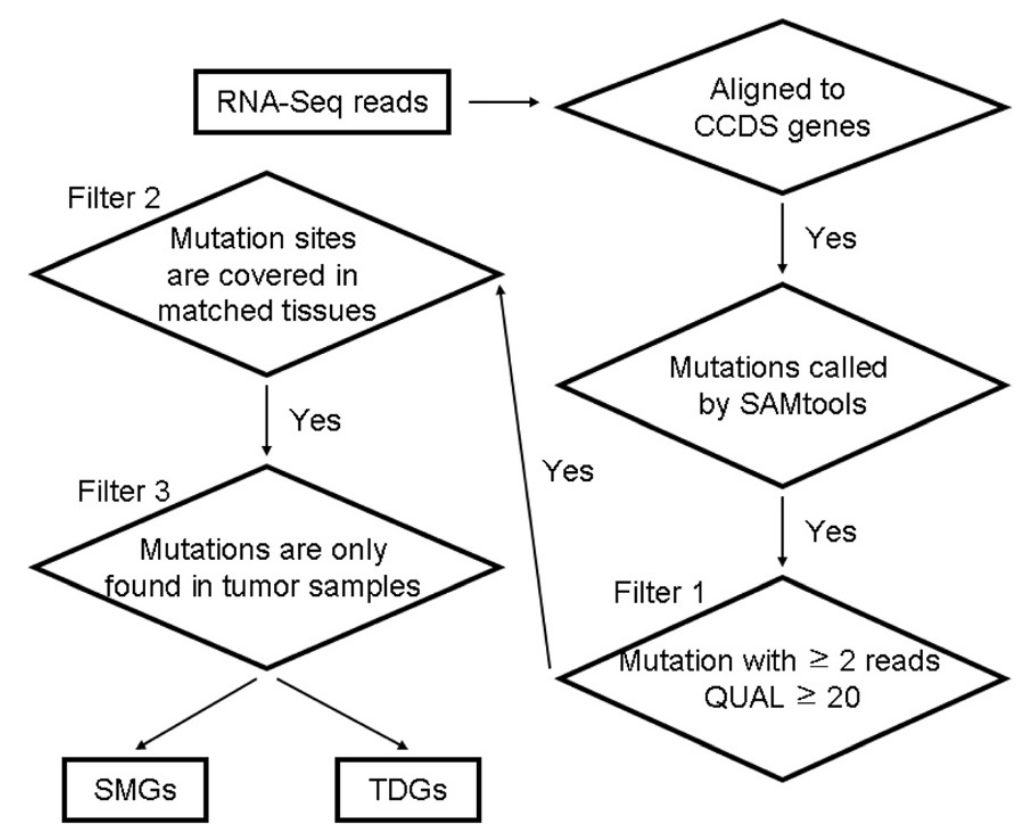

Figure 1 Flowchart of the bioinformatics pipeline. The input data is high-quality reads in which each base has a Q-score $\geq 20$. The output file is somatic mutations in tumor samples and was further feed into pipelines to identify significantly mutated genes (SMGs) and tumor-specific disruptive genes (TDGs). See Methods for more details.

test (FCPT) to generate a statistic [22], and the final $P$-value can be calculated according to a $X^{2}$ distribution with two times the number of categories as the degrees of freedom.

\section{Functional and pathway enrichment analysis}

To identify enriched gene functions, we extracted functional annotations from gene ontology (GO) [23] using bioconductor (http://www.bioconductor.org) package "org. Hs.eg.db". Then we used "topGO" package in R software [24] to perform hypergeometric tests. Kyoto Encyclopedia of Genes and Genomes pathway information [25,26] was extracted using bioconductor package "KEGG.db", and hypergeometric tests were used to identify enriched pathways.

\section{Differential gene expression}

To estimate gene expression abundance, we counted the number of reads that were aligned to each gene transcript. We then used bioconductor package "DESeq" [27] to identify differentially expressed genes. DESeq assumes a negative binomial distribution to estimate variance and mean for each group, and performs statistical test based on it. Multiple-testing was corrected by Benjamini and Hochberg procedure [28].

\section{Results}

\section{Read alignments and mutation spectrum}

Transcriptomes of paired normal and tumor tissues from two OSCC patients were sequenced by Applied
Biosystems SOLiD System, and $\sim 1,200$ million short reads were generated (200 million reads per sample), each 50-bp long [18]. A total of 187 million reads passed the filter and were aligned to 18,462 transcripts of UCSC CCDS genes using BFAST aligner (Table 1). To minimize possible sources of false positive in variant calling, we only kept $\sim 23$ million aligned reads (12.4\%) in total with a minimal mapping quality score of 30 . Then we used programs in SAMtools package to call variants. Due to the high error rate in massively parallel sequencing technique and short read alignment, we took extra care in variant identification and applied a series of stringent filters. Finally, we identified 144,400 somatic mutations in all samples and 70,472 of them passed all three filters (Table 2), and both patients showed a significant excess of somatic mutations in tumors $(P<2 \times$ $10^{-16}$ for patient 33 and $P=5.6 \times 10^{-6}$ for patient 51 , chisquare test), which is expected.

\section{Significantly mutated genes}

One distinguishable feature of tumor driver genes is the unexpectedly high somatic mutation rate, which leads to rapid accumulation of genetic aberrations and thus radical modification or disruption of gene functions. In hopes of finding tumor driver genes, we adopted an approach developed elsewhere [22] to identify significantly mutated genes (SMGs). Since the number of sequencing reads is highly variable among samples, we applied the pipeline to each sample separately. Significantly mutated 
Table 1 Summary statistics of whole transcriptome sequencing data used in this study

\begin{tabular}{|c|c|c|c|c|}
\hline & \multicolumn{2}{|l|}{ Patient 33} & \multicolumn{2}{|l|}{ Patient 51} \\
\hline & Normal & Tumor & Normal & Tumor \\
\hline Total reads & $229 \mathrm{M}$ & $256 M$ & $227 \mathrm{M}$ & $199 \mathrm{M}$ \\
\hline HQ reads $(\%)^{\mathrm{a}}$ & $68.9 \mathrm{M}(30.0)$ & $47.0 \mathrm{M}(18.3)$ & $53.1 \mathrm{M}(23.4)$ & $17.8 \mathrm{M}(9.0)$ \\
\hline HQ mapped $(\%)^{b}$ & $6.4 \mathrm{M}(9.3)$ & $4.4 \mathrm{M}(9.3)$ & $9.1 \mathrm{M}(17.1)$ & $3.4 \mathrm{M}$ (18.8) \\
\hline
\end{tabular}

${ }^{\mathrm{a}} \mathrm{HQ}$ reads high-quality reads in which each base has a Q-score $\geq 20$. Numbers in the brackets are percentages of high quality reads out of total reads.

${ }^{\mathrm{b}} \mathrm{HQ}$ mapped number of high quality reads of which mapping quality score $\geq 30$. Numbers in brackets are percentages of those mapped reads out of high quality reads.

genes in tumors were defined as genes that have $\geq 100$ base pairs covered by least three reads and have a FCPT $P$-value $<0.01$ in the two tumor samples but not in neither of the normal samples. In total, 515 significantly mutated genes were identified among 11,065 genes expressed in all samples (Additional file 1), and their average mutation rate $(0.0018$ per base $)$ is significantly higher than that of other genes $\left(0.0008\right.$ per base, $\left.P=2.89 \times 10^{-15}\right)$.

\section{Genes with disruptive mutations}

Genes with disruptive mutations in tumor samples are also of great interest, as they embrace the potential to radically change gene functions. To identify disruptive mutations, we annotated 70,472 somatic mutations identified above and searched for nonsynonymous mutations and indels. In total, 27,310 disruptive mutations were found in all samples (Table 2). Since our purpose was to identify tumor-specific disruptions, we only focused on tumor-specific disruptive genes (TDGs) which contain disruptive mutations in the two tumor samples but not in any normal samples. As a result, 156 genes were found as TDGs, of which six genes were also identified as SMGs.

\section{Gene ontology and pathway analysis}

We further performed gene ontology and pathway analysis on both SMG and TDG sets. Although no functional categories were enriched in TDGs, we found several enriched GO terms in SMGs (Table 3), including voltage-gated cation channel activity, intrinsic to membrane, integral to membrane, intrinsic to plasma membrane and integral to

Table 2 Summary statistics of variants or genes after each bioinformatic filter

\begin{tabular}{lllllll}
\hline & \multicolumn{2}{l}{ Patient 33 } & & \multicolumn{2}{c}{ Patient 51 } & \multirow{2}{*}{ Sum } \\
\cline { 2 - 3 } & Normal & Tumor & & Normal & Tumor & \\
\hline Raw & 44,185 & 41,645 & 41,970 & 16,600 & 144,400 \\
After filter 1 & 33,112 & 31,285 & & 34,164 & 13,020 & 111,581 \\
After filter 2 & 23,869 & 24,950 & 24,636 & 11,811 & 85,266 \\
After filter 3 & 20,175 & 21,246 & 20,861 & 8,190 & 70,472 \\
Coding & 9,367 & 10,295 & 8,870 & 3,476 & 32,008 \\
Disruptive & 8,058 & 8,827 & & 7,590 & 2,835 & 27,310 \\
Disruptive genes & 4,454 & 4,758 & 4,404 & 2,076 & 15,692 \\
\hline
\end{tabular}

plasma membrane. By interrogating KEGG pathways, we found SMGs were highly overrepresented in neuroactive ligand-receptor interaction, cell adhesion molecules (CAMs), and complement and coagulation cascades, while TDGs were enriched in steroid biosynthesis, ribosome, and aldosterone-regulated sodium reabsorption, at a relaxed $P$-value (0.1). The difference in functions and pathways between SMGs and TDGs suggests we captured different features of tumor in oral squamous cell carcinoma.

\section{Somatic mutations and gene expression}

To understand the potential consequence of SMGs and TDGs, especially in gene expression, we estimated gene expression abundance as the number of high-quality reads mapped to each gene, and used "DESeq" to identify genes with significantly differential expression between tumors and normal samples. In total, we found 41 differentially expressed genes (DEGs) with an adjusted $P<0.05$. Among them, five genes are SMGs, and one is TDG. The number of shared genes between DEGs and SMGs was highly unexpected $(P=0.002$, hypergeometric test), while no such pattern was observed for TDGs $(P=$ 0.07), indicating that SMGs may function through transcriptional regulation.

\section{Functional consequence of candidate genes}

There are six genes (TAF1L, ANKRA2, STOML1, PPP1R26, NUP37, and GTF2H5) identified in both SMGs and TDGs, which constitute the prioritized candidates for detailed functional dissection. Of them, five (TAF1L, ANKRA2, STOML1, PPP1R26, and NUP37) were reported in the Catalogue Of Somatic Mutations In Cancer (COSMIC, http://www.sanger.ac.uk/genetics/CGP/cosmic/, v62 release). However, somatic mutations in GTF2H5 identified in this study were not observed in 90 analyzed samples of the database. GTF2H5 encodes a 71-aa peptide, which is a subunit of the basal transcription factor TFIIH and functions in nucleotide excision repair and transcription [29]. To better understand the potential role of GTF2H5, we first examined its expression pattern (Figure 2), but no significant difference was observed between tumor and normal tissues $(P$-value $=1$ after Benjamini-Hochberg correction), nor was its downstream gene ERCC3 ( $P$-value $=1$ after Benjamini-Hochberg correction). We then predicted the effect of the amino acid changes in GTF2H5 using 
Table 3 Enriched GO and pathway categories

\begin{tabular}{|c|c|c|c|c|}
\hline Term & Description & Adjusted $P$ & Category $^{a}$ & GeneSet $^{\mathrm{b}}$ \\
\hline GO:0022843 & voltage-gated cation channel activity & 0.019 & MF & SMG \\
\hline GO:0031224 & intrinsic to membrane & 0.001 & $\mathrm{CC}$ & SMG \\
\hline GO:0016021 & integral to membrane & 0.001 & $\mathrm{CC}$ & SMG \\
\hline GO:0005887 & integral to plasma membrane & 0.009 & $\mathrm{CC}$ & SMG \\
\hline GO:0031226 & intrinsic to plasma membrane & 0.011 & CC & SMG \\
\hline HSA04080 & Neuroactive ligand-receptor interaction & 0.055 & KEGG & SMG \\
\hline HSA04514 & Cell adhesion molecules (CAMs) & 0.052 & KEGG & SMG \\
\hline HSA04610 & Complement and coagulation cascades & 0.052 & KEGG & SMG \\
\hline HSA00100 & Steroid biosynthesis & 0.110 & KEGG & TDG \\
\hline HSA03010 & Ribosome & 0.110 & KEGG & TDG \\
\hline HSA04960 & Aldosterone-regulated sodium reabsorption & 0.110 & KEGG & TDG \\
\hline
\end{tabular}

${ }^{a} M F$ molecular function term in GO, CC cellular component term in GO, KEGG KEGG pathway terms.

${ }^{b} S M G$ significantly mutated genes, TDG tumor-specific disruptive genes (see main text for details).

SIFT tool [30], however, no intolerant changes were found among these disruptive mutations. Since the major determinant in oral cancers is the accumulation of genomic instability [31], and no obvious evidence was observed in radical mutations or gene expression, it is possible that the excess of somatic mutations in GTF2H5 may influence post-transcriptional regulation and correlate with tumor development.

\section{Discussion}

It is well accepted that the accumulation of multiple genetic events in different genes and molecular pathways is the main cause of OSCC evolution [3,32]. Previous studies have identified various types of genetic aberrations in OSCCs and oral dysplasias, the precursors of

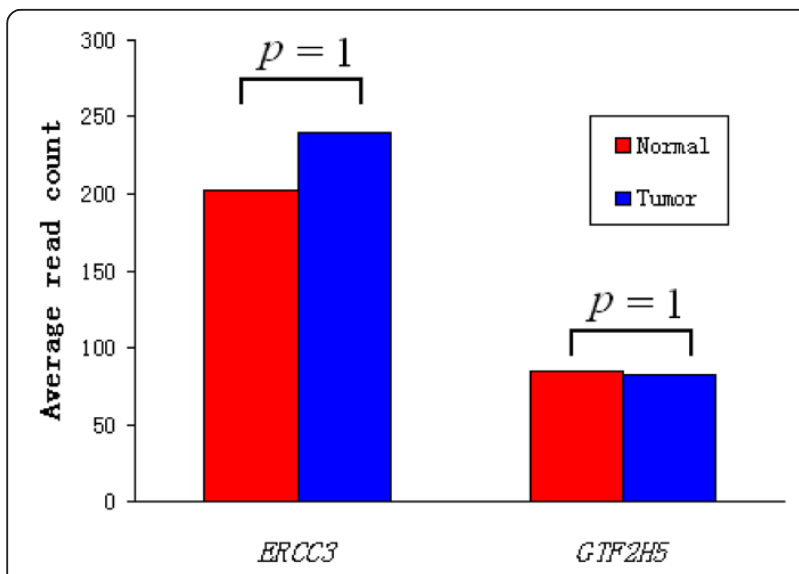

Figure 2 Gene expression pattern for GTF2H5 and its downstream gene ERCC3. The expression level was estimated by RSEM, which counts the number of reads mapped to each transcript and normalized by the total number of reads in each sample. No significant difference was found between tumor and normal tissues for each gene.
OSCCs, including somatic mutations in the D-loop of mtDNA sequence [33] and in exons nine and 20 of Phosphatidylinositol 3-kinase gene (PI3K) [34], common deletions on chromosome $3 \mathrm{p}$ such as the $3 \mathrm{p} 14$ locus that harbors FHIT (Fragile Histidine Triad) [35-37], as well as gene copy number increases in certain oncogenes such as EGFR and CCND1 [31,38,39]. Evidence from microarray studies has also revealed differentially expressed genes in oral cavity tumors [40-44], suggesting multiple dimensions of genetic aberrations contributing to OSCC development. Here, we presented a whole transcriptome analysis to identify exonic somatic mutations in two OSCC samples. To overcome the small sample size, we have developed a stringent bioinformatic pipeline with multiple filters to reduce false positives. In total, we have identified 515 SMGs which were significantly mutated, and 156 TDGs with disruptive mutations in both tumor samples. We also measured gene expression and found SMGs were enriched in differentially expressed genes, implying that the accumulation of genetic aberrations may regulate corresponding gene expression and further affect tumor evolution.

Five of six genes identified in both SMGs and TDGs are known driver genes in COSMIC database, and the remaining gene GTF2H5 stimulates the ATPase activity of $E R C C 3$, a nucleotide excision repair gene, to trigger DNA opening during DNA repair. Since genes involved in DNA repair functions are commonly associated with oral cancer [45-47], it is very likely that GTF2H5 is also related to carcinogenesis. Collectively, these observations indicate our bioinformatic pipeline has substantial power to identify tumor-related genes.

Of 515 SMGs, several membrane-related GO terms were enriched, including intrinsic to membrane, integral to membrane, intrinsic to plasma membrane and integral to plasma membrane. Interestingly, the original study found that the term of intrinsic to plasma membrane was 
enriched in mis-regulated genes in tumor samples [18], suggesting that disruption or mis-expression of genes related to plasma membrane may be involved in tumor development. We also found six tumor related genes, TUSC2, TP53I3, TSSC4, RAB23, RAB39A, and ERG, which function as either tumor suppressor genes or oncogenes. Additionally, we identified $F G F 2$, a fibroblast growth factor in the FGF signaling pathway, which was reported to be important in OSCCs [3]. Another pathway potentially associated with OSCC is the cell adhesion molecules (CAMs), which is also enriched in SMGs. CAMs are essential component to maintain the structure of stratified squamous epithelium and a critical mediator of tumor progression in OSCC $[48,49]$, and mis-expression or dysfunction of CAMs are shown to contribute to malignant tumors [48]. The excess of CAMs in SMGs further suggests the critical role of the CAM pathway in OSCCs. Again, cell adhesion was found to be enriched in misregulated genes in the original study [18], confirming that tumor development may involve both mis-expression and dysfunction of CAMs.

Tumor driver genes are normally considered as with high somatic mutation rate, thus 156 TDGs identified without information from mutation rate are intriguing. Besides six genes also identified as SMGs, we also found that 57 (37\%) TDGs significantly mutated in one tumor sample but not in the other tumor sample. Considering that only two patients were used in this study and a large proportion of TDGs were significantly mutated in only one sample, it is possible that some TDGs are in fact SMGs, but failed to be identified here due to the small sample size. We thus suggest that screening TDGs may be an alternative way to identify candidate cancer driver genes when sample size is limited.

Although a few pioneer studies demonstrated that RNA-Seq is suitable for identifying somatic mutations [14-17,50,51], there is a concern that RNA-Seq is prone to error [15] and may generate a high false discovery rate due to incorrect alignment of reads, sequencing errors or extremely high or low read coverage. To minimize the false positive rate, we have applied a series of stringent filters. First, we only used reads in which each base has a Q-score $\geq 20$, which reduces the influence of sequencing errors. Next we filtered out read alignments with a mapping quality lower than 30 , which avoids reads mapped to multiple locations alignments with low similarity. Then we required each qualified variant must have a read depth between three and 500. Our strategy to identify somatic mutations also automatically removed the effect of systematically incorrect alignments which present in both tumor and matched samples. Hence we believe that somatic mutations identified in this study provide a substantial list of candidates for biomarker development. However, it should also be noted that we only focused on exonic regions captured by RNA-Seq, somatic mutations in regulatory regions will not be identified here, therefore out list also presents a portion of somatic mutations in OSCCs.

\section{Conclusions}

In this study, we have developed a stringent bioinformatic pipeline to identify somatic mutations in tumors and applied it to two OSCC paired samples. By using multiple filters and calling candidate disruptive genes through two different ways, we minimized both false positives and false negatives due to the small sample size. The resulting candidate genes with both statistical and biological significance would help understand the molecular mechanism of OSCC and develop clinical biomarkers and drug targets.

\section{Additional file}

Additional file 1: List of significantly mutated genes (SMGs) and tumor-specific disruptive genes (TDGs).

\section{Competing interests}

The authors declare no conflict of interest.

Authors' contributions

QZ and SS conceived the project. QZ, JZ, and HJ carried out the data analysis. QZ and SS drafted the manuscript. All authors read and approved the final manuscript.

\section{Acknowledgements}

We thank Dr. Yinglei Lai , Dr. Luiz De Marco and Dr. Yuji Miyazaki for helpful comments on this manuscript. This work was supported by the Introduction of Innovative R\&D Team Program of Guangdong Province (China, NO. 2009010029).

\section{Author details}

'Department of Human Evolutionary Biology, Harvard University, Cambridge, MA 02138, USA. ²Department of Surgery, Shanghai Institute of Digestive Surgery, Rui Jin Hospital, Shanghai Jiaotong University, School of Medicine, No.197 Ruijin er Road, Shanghai, P.R. China. ${ }^{3} \mathrm{HYK}$ High-throughput

Biotechnology Institute, 4th Floor, Building No.11, Software Park, 2nd Central Keji Rd, Hi-Tech Industrial Park, Shenzhen, China.

Received: 18 April 2013 Accepted: 26 August 2013

Published: 4 September 2013

\section{References}

1. Parkin DM, Bray F, Ferlay J, Pisani P: Global cancer statistics, 2002. CA Cancer J Clin 2005, 55(2):74-108.

2. Jemal A, Siegel R, Ward E, Hao Y, Xu J, Thun MJ: Cancer statistics, 2009. CA Cancer J Clin 2009, 59(4):225-249.

3. Gibb EA, Enfield KS, Tsui IF, Chari R, Lam S, Alvarez CE, Lam WL: Deciphering squamous cell carcinoma using multidimensional genomic approaches. J Skin Cancer 2011, 2011:541405.

4. Vogelstein B, Kinzler KW: Cancer genes and the pathways they control. Nat Med 2004, 10(8):789-799.

5. Greenman C, Stephens P, Smith R, Dalgliesh GL, Hunter C, Bignell G, Davies $\mathrm{H}$, Teague J, Butler A, Stevens C, et al: Patterns of somatic mutation in human cancer genomes. Nature 2007, 446(7132):153-158.

6. Jones S, Zhang X, Parsons DW, Lin JC, Leary RJ, Angenendt P, Mankoo P, Carter $\mathrm{H}$, Kamiyama $\mathrm{H}$, Jimeno $\mathrm{A}$, et al: Core signaling pathways in human pancreatic cancers revealed by global genomic analyses. Science 2008, 321(5897):1801-1806.

7. Sjoblom T, Jones S, Wood LD, Parsons DW, Lin J, Barber TD, Mandelker D, Leary RJ, Ptak J, Silliman N, et al: The consensus coding sequences of human breast and colorectal cancers. Science 2006, 314(5797):268-274. 
8. Wood LD, Parsons DW, Jones S, Lin J, Sjoblom T, Leary RJ, Shen D, Boca SM, Barber T, Ptak J, et al: The genomic landscapes of human breast and colorectal cancers. Science 2007, 318(5853):1108-1113.

9. Wang Z, Gerstein M, Snyder M: RNA-Seq: a revolutionary tool for transcriptomics. Nat Rev Genet 2009, 10(1):57-63.

10. Mortazavi A, Williams BA, McCue K, Schaeffer L, Wold B: Mapping and quantifying mammalian transcriptomes by RNA-Seq. Nat Methods 2008, 5(7):621-628.

11. Zhang LQ, Cheranova D, Gibson M, Ding S, Heruth DP, Fang D, Ye SQ: RNAseq Reveals Novel Transcriptome of Genes and Their Isoforms in Human Pulmonary Microvascular Endothelial Cells Treated with Thrombin. PLoS One 2012, 7(2):e31229.

12. Gregg C, Zhang J, Butler JE, Haig D, Dulac C: Sex-specific parent-of-origin allelic expression in the mouse brain. Science 2010, 329(5992):682-685.

13. Gregg C, Zhang J, Weissbourd B, Luo S, Schroth GP, Haig D, Dulac C: Highresolution analysis of parent-of-origin allelic expression in the mouse brain. Science 2010, 329(5992):643-648.

14. Cloonan N, Forrest AR, Kolle G, Gardiner BB, Faulkner GJ, Brown MK, Taylor DF, Steptoe AL, Wani S, Bethel G, et al: Stem cell transcriptome profiling via massive-scale mRNA sequencing. Nat Methods 2008, 5(7):613-619.

15. Cirulli ET, Singh A, Shianna KV, Ge D, Smith JP, Maia JM, Heinzen EL, Goedert JJ, Goldstein DB: Screening the human exome: a comparison of whole genome and whole transcriptome sequencing. Genome Biol 2010, 11(5):R57.

16. Kridel R, Meissner B, Rogic S, Boyle M, Telenius A, Woolcock B, Gunawardana J, Jenkins C, Cochrane C, Ben-Neriah S, et al: Whole transcriptome sequencing reveals recurrent NOTCH1 mutations in mantle cell lymphoma. Blood 2012 119(9):1963-1971.

17. Canovas A, Rincon G, Islas-Trejo A, Wickramasinghe S, Medrano JF: SNP discovery in the bovine milk transcriptome using RNA-Seq technology. Mamm Genome 2010, 21(11-12):592-598.

18. Tuch BB, Laborde RR, Xu X, Gu J, Chung CB, Monighetti CK, Stanley SJ, Olsen KD, Kasperbauer $\mathrm{L}$, Moore EJ, et al: Tumor transcriptome sequencing reveals allelic expression imbalances associated with copy number alterations. PLoS One 2010, 5(2):e9317.

19. Homer N, Merriman B, Nelson SF: BFAST: an alignment tool for large scale genome resequencing. PLoS One 2009, 4(11):e7767.

20. Li H, Handsaker B, Wysoker A, Fennell T, Ruan J, Homer N, Marth G, Abecasis G, Durbin R: The Sequence Alignment/Map format and SAMtools. Bioinformatics 2009, 25(16):2078-2079.

21. Sherry ST, Ward MH, Kholodov M, Baker J, Phan L, Smigielski EM, Sirotkin K: dbSNP: the NCBI database of genetic variation. Nucleic Acids Res 2001, 29(1):308-311.

22. Dees ND, Zhang Q, Kandoth C, Wendl MC, Schierding W, Koboldt DC, Mooney TB, Callaway MB, Dooling D, Mardis ER, et al: MuSiC: identifying mutational significance in cancer genomes. Genome Res 2012, 22(8):1589-1598.

23. Ashburner M, Ball CA, Blake JA, Botstein D, Butler H, Cherry JM, Davis AP, Dolinski K, Dwight SS, Eppig JT, et al: Gene ontology: tool for the unification of biology, The Gene Ontology Consortium. Nat Genet 2000, 25(1):25-29.

24. Alexa A, Rahnenfuhrer J, Lengauer T: Improved scoring of functional groups from gene expression data by decorrelating GO graph structure. Bioinformatics 2006, 22(13):1600-1607.

25. Kanehisa M, Goto S: KEGG: kyoto encyclopedia of genes and genomes. Nucleic Acids Res 2000, 28(1):27-30.

26. Kanehisa M, Goto S, Sato Y, Furumichi M, Tanabe M: KEGG for integration and interpretation of large-scale molecular data sets. Nucleic Acids Res 2012, 40(Database issue):D109-D114.

27. Anders S, Huber W: Differential expression analysis for sequence count data. Genome Biol 2010, 11(10):R106

28. Benjamini $Y$, Hochberg $Y$ : Controlling the false discovery rate: a practical and powerful approach to multiple testing. J R Stat SoC B 1995, 57(1):12.

29. Moslehi R, Kumar A, Mills JL, Ambroggio X, Signore C, Dzutsev A: Phenotype-specific adverse effects of XPD mutations on human prenatal development implicate impairment of TFIIH-mediated functions in placenta. Eur J Hum Genet 2012, 20(6):626-631.

30. Kumar P, Henikoff S, Ng PC: Predicting the effects of coding nonsynonymous variants on protein function using the SIFT algorithm. Nat Protoc 2009, 4(7):1073-1081.

31. Garnis C, Chari R, Buys TP, Zhang L, Ng RT, Rosin MP, Lam WL: Genomic imbalances in precancerous tissues signal oral cancer risk. Mol Cancer $2009,8: 50$
32. Mao L, Hong WK, Papadimitrakopoulou VA: Focus on head and neck cancer. Cancer Cell 2004, 5(4):311-316.

33. Liu SA, Jiang RS, Chen FJ, Wang WY, Lin JC: Somatic mutations in the D-loop of mitochondrial DNA in oral squamous cell carcinoma. Eur Arch Otorhinolaryngol 2012, 269(6):1665-1670.

34. Kozaki K, Imoto I, Pimkhaokham A, Hasegawa S, Tsuda H, Omura K, Inazawa J: PIK3CA mutation is an oncogenic aberration at advanced stages of oral squamous cell carcinoma. Cancer Sci 2006, 97(12):1351-1358.

35. Califano J, van der Riet P, Westra W, Nawroz H, Clayman G, Piantadosi S, Corio R, Lee D, Greenberg B, Koch W, et al: Genetic progression model for head and neck cancer: implications for field cancerization. Cancer Res 1996, 56(11):2488-2492.

36. Tsui IF, Rosin MP, Zhang L, Ng RT, Lam WL: Multiple aberrations of chromosome $3 p$ detected in oral premalignant lesions. Cancer Prev Res (Phila) 2008, 1(6):424-429.

37. Rosin MP, Cheng X, Poh C, Lam WL, Huang Y, Lovas J, Berean K, Epstein JB, Priddy $R$, Le ND, et al: Use of allelic loss to predict malignant risk for lowgrade oral epithelial dysplasia. Clin Cancer Res 2000, 6(2):357-362

38. Tsui IF, Poh CF, Garnis C, Rosin MP, Zhang L, Lam WL: Multiple pathways in the FGF signaling network are frequently deregulated by gene amplification in oral dysplasias. Int J Cancer 2009, 125(9):2219-2228.

39. Myllykangas S, Bohling T, Knuutila S: Specificity, selection and significance of gene amplifications in cancer. Semin Cancer Biol 2007, 17(1):42-55.

40. Leethanakul C, Patel V, Gillespie J, Pallente M, Ensley JF, Koontongkaew S, Liotta LA, Emmert-Buck M, Gutkind JS: Distinct pattern of expression of differentiation and growth-related genes in squamous cell carcinomas of the head and neck revealed by the use of laser capture microdissection and cDNA arrays. Oncogene 2000, 19(28):3220-3224.

41. Alevizos I, Mahadevappa M, Zhang X, Ohyama H, Kohno Y, Posner M, Gallagher GT, Varvares M, Cohen D, Kim D, et al: Oral cancer in vivo gene expression profiling assisted by laser capture microdissection and microarray analysis. Oncogene 2001, 20(43):6196-6204.

42. Belbin TJ, Singh B, Barber I, Socci N, Wenig B, Smith R, Prystowsky MB, Childs G: Molecular classification of head and neck squamous cell carcinoma using CDNA microarrays. Cancer Res 2002, 62(4):1184-1190.

43. Al Moustafa AE, Alaoui-Jamali MA, Batist G, Hernandez-Perez M, Serruya C, Alpert $L$, Black MJ, Sladek R, Foulkes WD: Identification of genes associated with head and neck carcinogenesis by cDNA microarray comparison between matched primary normal epithelial and squamous carcinoma cells. Oncogene 2002, 21(17):2634-2640.

44. $\mathrm{Yu}$ YH, Kuo HK, Chang KW: The evolving transcriptome of head and neck squamous cell carcinoma: a systematic review. PLoS One 2008, 3(9):e3215.

45. Zavras Al, Yoon AJ, Chen MK, Lin CW, Yang SF: Association between polymorphisms of DNA repair gene ERCC5 and oral squamous cell carcinoma. Oral Surg Oral Med Oral Pathol Oral Radiol 2012, 114(5):624-629.

46. Mukherjee S, Bhowmik AD, Roychoudhury P, Mukhopadhyay K, Ray JG, Chaudhuri K: Association of XRCC1, XRCC3, and NAT2 polymorphisms with the risk of oral submucous fibrosis among eastern Indian population. J Oral Pathol Med 2012, 41(4):292-302.

47. Vaezi A, Wang X, Buch S, Gooding W, Wang L, Seethala RR, Weaver DT, D'Andrea AD, Argiris A, Romkes M, et al: XPF expression correlates with clinical outcome in squamous cell carcinoma of the head and neck. Clin Cancer Res 2011, 17(16):5513-5522.

48. Thomas GJ, Speight PM: Cell adhesion molecules and oral cancer. Crit Rev Oral Biol Med 2001, 12(6):479-498.

49. Hung SC, Wu IH, Hsue SS, Liao CH, Wang HC, Chuang PH, Sung SY, Hsieh $\mathrm{CL}$ : Targeting I1 cell adhesion molecule using lentivirus-mediated short hairpin RNA interference reverses aggressiveness of oral squamous cell carcinoma. Mol Pharm 2010, 7(6):2312-2323.

50. Chepelev I, Wei G, Tang Q, Zhao K: Detection of single nucleotide variations in expressed exons of the human genome using RNA-Seq. Nucleic Acids Res 2009, 37(16):e106.

51. Morin R, Bainbridge M, Fejes A, Hirst M, Krzywinski M, Pugh T, McDonald H, Varhol R, Jones S, Marra M: Profiling the HeLa S3 transcriptome using randomly primed CDNA and massively parallel short-read sequencing. Biotechniques 2008, 45(1):81-94.

doi:10.1186/1755-8794-6-28

Cite this article as: Zhang et al:: Whole transcriptome sequencing identifies tumor-specific mutations in human oral squamous cell carcinoma. BMC Medical Genomics 2013 6:28. 